\title{
Analysis of Compactly Supported Transformations for Landmark-based Image Registration
}

\author{
Roberto Cavoretto and Alessandra De Rossi ${ }^{*}$ \\ Department of Mathematics "G. Peano”, University of Torino, via Carlo Alberto 10, I-10123 Torino, Italy
}

Received: 23 Feb. 2013, Revised: 21 Jun. 2013, Accepted: 22 Jun. 2013

Published online: 1 Nov. 2013

\begin{abstract}
In this paper we consider landmark-based image registration using radial basis function interpolation schemes. More precisely, we analyze some landmark-based image transformations using compactly supported radial basis functions such as Wendland's, Wu's, and Gneiting's functions. Comparisons of interpolation techniques are performed and numerical experiments show differences in accuracy and smoothness of them in some test cases. Finally, a real-life case with medical images is considered.
\end{abstract}

Keywords: Scattered data interpolation, radial basis functions, Wendland's functions, elastic registration.

\section{Introduction}

Image registration is an important challenging topic in image processing and information sciences. It consists mainly in finding a suitable transformation between two images (or image data), called source and target images, taken either at different times or from different sensors or viewpoints. The scope is to determine a transformation such that the transformed version of the source image is similar to the target one. There is a large number of applications demanding image registration, including astronomy, biology, computer vision, genetics, physics, medicine, robotics, to name a few. For an overview, see e.g. $[8,9,12-14,17-19,22,23,26]$ and references therein. In medicine, for example, registration is required for combining different modalities (X-ray, computer tomography (CT), magnetic resonance imaging (MRI) and positron emission tomography (PET) images, for instance), monitoring of diseases, treatment validation, comparison of the patient's data with anatomical atlases, and radiation therapy. In particular, the landmark-based image registration process is based on two finite sets of landmarks, i.e. sparse data points located on images, usually not uniformly distributed, where each landmark of the source image has to be mapped onto the corresponding landmark of the target image (see $[17,18,22])$. The landmark-based registration problem can be formulated in the context of multivariate scattered data interpolation, and solved by different techniques, among which radial basis functions (RBFs) play a preminent role (see, e.g., [7, 24]). The use of RBF transformations, in particular of the thin plate splines, for point-based image registration was first proposed by Bookstein [3], and it is still common (see [20,21] and the software package MIPAV [16]).

Since using globally supported RBFs, as for example the thin plate spline or the Gaussian, a single landmark pair change may influence the whole registration result, in the last decade several methods have been presented to circumvent this disadvantage, such as Wendland's compactly supported radial basis functions (CSRBFs) [10], elastic body splines (EBSs) [15], the modified inverse distance weighted method (IDWM) [4, 5], and a spline method [1, 2]. These interpolation techniques, giving rise to compactly supported or local mappings, handle well images locally deformed. Moreover, they are in general stable and the computational effort to determine transformations is low and, therefore, also a large number of landmarks can be used.

In this paper we focus on properties and performances of CSRBFs. In particular, we consider in this context compactly supported transformations given by Wendland's, Wu's, and Gneiting's functions. These methods are also compared with Gaussians and thin plate splines, which are globally supported but are still among the most widely used methods in applications. Numerical experiments point out differences in accuracy and

\footnotetext{
*Corresponding author e-mail: alessandra.derossi@unito.it
} 
smoothness of the considered methods. We think that the comparison can be useful to users in the choice of the appropriate transformation for their scopes.

The paper is organized as follows. Section 2 introduces the landmark-based registration problem. In Section 3 we briefly recall radial basis functions, like Gaussians, multiquadrics, inverse multiquadrics and thin plate splines to construct globally supported transformations. In Section 4 Wendland's, Wu's and Gneiting's functions are presented to define compactly supported transformations. Finally, Section 5 contains several numerical results obtained in some test and real examples: special emphasis is devoted to comparing accuracy of CSRBFs schemes.

\section{The landmark-based image registration problem}

For simplicity, in this section and in the following, we limit the presentation to the $2 D$ case, but all definitions can be easily extended to the $3 D$ one.

Let $\mathscr{S}_{N}=\left\{\mathbf{x}_{j} \in \mathbb{R}^{2}, j=1,2, \ldots, N\right\}$ be a given set of landmarks in the source image $S$ and let $\mathscr{T}_{N}=\left\{\mathbf{t}_{j} \in \mathbb{R}^{2}, j=1,2, \ldots, N\right\}$ be the given set of corresponding landmarks in the target image $T$. The registration problem reads as follows.

Problem 1.Let the landmark sets $\mathscr{S}_{N}$ and $\mathscr{T}_{N}$ be given. Find a transformation $\mathbf{F}: \mathbb{R}^{2} \rightarrow \mathbb{R}^{2}$ within a suitable space $\mathscr{F}$ of admissible functions, such that

$\mathbf{F}\left(\mathbf{x}_{j}\right)=\mathbf{t}_{j}, \quad j=1,2, \ldots, N$.

Each coordinate $F_{k}$ of the transformation function is calculated separately, i.e. the interpolation problem $F_{k}: \mathbb{R}^{2} \rightarrow \mathbb{R}$ is solved for each $k=1,2$, with the corresponding conditions

$F_{k}\left(\mathbf{x}_{j}\right)=t_{j k}, \quad j=1,2, \ldots, N$.

In order to have a class of basis functions that generate non-singular interpolation matrices for any set of distinct points, we introduce the concept of strictly positive definite functions [7]. We suppose that the interpolant $F_{k}: \mathbb{R}^{2} \rightarrow \mathbb{R}$ has the form

$$
F_{k}(\mathbf{x})=\sum_{j=1}^{N} c_{j k} \Psi\left(\mathbf{x}-\mathbf{x}_{j}\right),
$$

$c_{j k}$ being the coefficients to be found. A necessary condition to have unique solvability of the interpolation problem (2) is given by the following result [7].

Theorem 1.The interpolation problem (2), where $F_{k}$ is of the form (3), has a unique solution if the function $\Psi$ is strictly positive definite on $\mathbb{R}^{2}$, that is

$\sum_{i=1}^{N} \sum_{j=1}^{N} c_{i} c_{j} \Psi\left(\boldsymbol{x}_{i}-\boldsymbol{x}_{j}\right)>0$ for any $N$ pairwise different points $\boldsymbol{x}_{1}, \boldsymbol{x}_{2}, \ldots, \boldsymbol{x}_{N} \in \mathbb{R}^{2}$, and $\boldsymbol{c}=\left[c_{1}, c_{2}, \ldots, c_{N}\right]^{T} \in \mathbb{R}^{N}, \boldsymbol{c} \neq \mathbf{0}$.

Moreover, we remark that Theorem 1 is also satisfied for a strictly conditionally positive definite function $\Psi$ of order $v$ if the quadratic form (4) holds and

$$
\sum_{i=1}^{N} c_{i} p\left(\mathbf{x}_{i}\right)=0
$$

for any polynomial $p$ of degree at most $v-1$.

\section{Radial basis functions}

In this section we consider globally supported radial basis functions, which are well-known in the field of approximation theory and scattered data interpolation (see [7, 24]), and widely used in landmark-based image registration as well (see, e.g., [18] and references therein).

Using radial basis functions, the general coordinate $F_{k}(\mathbf{x}), k=1,2$ of the transformation function is assumed to have the form

$$
F_{k}(\mathbf{x})=\sum_{j=1}^{N} c_{j k} \Phi\left(\left\|\mathbf{x}-\mathbf{x}_{j}\right\|_{2}\right)+\sum_{i=1}^{U} b_{i k} \pi_{i k}(\mathbf{x}),
$$

where $\Phi\left(\left\|\mathbf{x}-\mathbf{x}_{j}\right\|_{2}\right)$ is a radial basis function depending only on the Euclidean distance $r=\left\|\mathbf{x}-\mathbf{x}_{j}\right\|_{2}$, and $c_{j k}$ and $b_{i k}$ are coefficients to be determined. The space $\mathscr{P}_{v-1}^{2} \equiv$ $\mathscr{P}_{v-1}\left(\mathbb{R}^{2}\right)=\operatorname{span}\left\{\pi_{i k}\right\}_{i=1}^{U}$, where the $\pi_{i k}$ are a basis of polynomials up to degree $v-1$, has dimension $U=(2+v-1) ! /(2 !(v-1) !)$, which must be lower than $N$. Therefore, in order to compute the coefficients $\mathbf{c}=\left(c_{1 k}, c_{2 k}, \ldots, c_{N k}\right)^{T}$ and $\mathbf{b}=\left(b_{1 k}, b_{2 k}, \ldots, b_{U k}\right)^{T}$ in (5), it is required to solve the following system of linear equations

$$
\left\{\begin{aligned}
\mathbf{M c}+\mathbf{Q b} & =\mathbf{t}, \\
\mathbf{Q}^{T} \mathbf{c} & =\mathbf{0},
\end{aligned}\right.
$$

where $\mathbf{M}=\left\{\Phi\left(\left\|\mathbf{x}_{i}-\mathbf{x}_{j}\right\|_{2}\right)\right\}$ is a $N \times N$ matrix, $Q=\left\{\pi_{i k}\left(\mathbf{x}_{j}\right)\right\}$ is a $N \times U$ matrix, and $\mathbf{t}$ denotes the column vector of the $k$-th coordinate of the target point-landmarks $\mathbf{t}_{j}$ corresponding to the image point-landmarks $\mathbf{x}_{j}$. Equations (6) are obtained by requiring that $\mathbf{F}$ satisfies the interpolation conditions (1) and the side conditions $\sum_{j=1}^{N} c_{j k} \pi_{i k}\left(\mathbf{x}_{j}\right)=0$, for $i=1,2, \ldots, U$, i.e. $\mathbf{Q}^{T} \mathbf{c}=\mathbf{0}$.

The most popular choices for $\Phi$ in landmark-based registration are

$$
\begin{aligned}
& \Phi(r)=r^{2} \log r, \quad \text { (thin plate spline) } \\
& \Phi(r)=\mathrm{e}^{-\alpha^{2} r^{2}}, \quad \text { (Gaussian) } \\
& \Phi(r)=\left(r^{2}+\gamma^{2}\right)^{\mu / 2}, \quad \text { (generalized multiquadric) }
\end{aligned}
$$

where $\alpha, \gamma \in \mathbb{R}^{+}$and $\mu \in \mathbb{Z}$. The Gaussian (G) and the inverse multiquadric (IMQ), which occurs for $\mu<0$ in 
the generalized multiquadric function, are strictly positive definite functions, whereas the thin plate spline (TPS) and the multiquadric (MQ), i.e. for $\mu>0$ in the generalized multiquadric function, are strictly conditionally positive definite functions of order $\mu$. The addition of a polynomial term of a certain order along with side conditions, in order to guarantee existence and uniqueness of the solution in the linear equation system (6), is required only for strictly conditionally positive definite functions. It allows us to have a nonsingular interpolation matrix. For thin plate spline the order of the polynomial is one and for multiquadric depends on the exponent $\mu$, the minimal degree being $v=\mu-1$. Polynomials have global support and therefore the polynomial part influences globally the registration result.

An important feature of some radial basis functions is the presence of a shape parameter, which allows us to control their influence on the registration result [10]. On the other hand this property could be seen as a drawback, because the user needs to give the parameter value. Also for this reason the thin plate spline is usually considered more suitable than other RBFs for image registration, since this process of image registration must sometimes be automatic.

The thin plate spline seems to be preferable to other radial basis functions for image registration also for other reasons. The thin plate spline minimizes a functional which represents the bending energy of a thin plate separately for each component $F_{k}, k=1,2$, of the transformation $\mathbf{F}$. Thus, the functional $J(\mathbf{F})$ can be separated into a sum of similar functionals that only depend on one component $F_{k}$ of $\mathbf{F}$, and the problem of finding $\mathbf{F}$ can be decomposed into two problems.

Note that the interpolation matrices generated by radial basis functions are dense, since they are globally supported, and ill-conditioned [7], especially those generated by Gaussian. Ill-conditioning could happen even if it is required to interpolate a relatively small number of landmarks, since the landmarks may be very close to each other.

Another possible disadvantage of the use of RBFs is given by the number of floating-point operations, which can be very time consuming, especially if the number of landmarks is large or the registration of $3 \mathrm{D}$ images is needed.

\section{Compactly supported radial basis functions}

In this section we consider the most popular families of CSRBFs such as Wendland's, Wu's and Gneiting's functions (see [7, 11, 24, 25]). Wendland's functions have been introduced in image registration context with the motivation that their influence around a landmark is limited, in 2D and 3D images on a circle or a sphere, respectively [10]. This property allows us the registration of medical images where changes occur only locally.
Here we propose also the use of Wu's and Gneiting's functions (see [6]).

\subsection{Wendland's functions}

Wendland's functions are obtained by using the truncated power function $\varphi_{s}(r)=(1-r)_{+}^{s}$ (where $(x)_{+}$is defined as $x$ for $x>0$ and 0 for $x \leq 0)$, which is strictly positive definite and radial on $\mathbb{R}^{m}$ for $s \geq\lfloor m / 2\rfloor+1$, and repeatedly applying the operator $\mathscr{I}$ given by $(\mathscr{I} \varphi)(r)=\int_{r}^{\infty} t \varphi(t) d t, r \geq 0$ (see, e.g., [24]). Then, Wendland's functions are given by

$$
\varphi_{m, h}=\mathscr{I}^{h} \varphi_{\lfloor m / 2\rfloor+h+1},
$$

Specifically, they are all supported on the interval $[0,1]$ and have a polynomial representation there. In addition to this, the following theorem states that any other compactly supported $C^{2 h}$ polynomial function that is strictly positive and radial on $\mathbb{R}^{m}$ will not have a smaller polynomial degree. Finally, they have minimal degree with respect to a given space dimension $m$ and smoothness $2 h$ [24].

Theorem 2.The functions $\varphi_{m, h}$ are strictly positive definite and radial on $\mathbb{R}^{m}$ and are of the form

$$
\varphi_{m, h}(r)= \begin{cases}p_{m, h}(r), & r \in[0,1] \\ 0, & r>1,\end{cases}
$$

with a univariate polynomial $p_{m, h}$ of degree $\lfloor m / 2\rfloor+3 h+1$. Moreover, $\varphi \in C^{2 h}(\mathbb{R})$ are unique up to a constant factor, and the polynomial degree is minimal for given space dimension $m$ and smoothness $2 h$.

Though there exist recursive formulas to compute the functions $\varphi_{m, h}$ for all $m$ and $h$, here for convenience we only give explicit forms of $\varphi_{m, h}$, for $h=0,1,2,3$.

Theorem 3.The functions $\varphi_{m, h}, h=0,1,2,3$, have the form

$$
\begin{aligned}
& \varphi_{m, 0}(r) \doteq(1-r)_{+}^{s}, \\
& \varphi_{m, 1}(r) \doteq(1-r)_{+}^{s+1}[(s+1) r+1] \\
& \varphi_{m, 2}(r) \doteq(1-r)_{+}^{s+2}\left[\left(s^{2}+4 s+3\right) r^{2}+(3 s+6) r+3\right] \\
& \varphi_{m, 3}(r) \doteq(1-r)_{+}^{s+3}\left[\left(s^{3}+9 s^{2}+23 s+15\right) r^{3}+\right. \\
& \left.+\left(6 s^{2}+36 s+45\right) r^{2}+(15 s+45) r+15\right]
\end{aligned}
$$

where $s=\lfloor m / 2\rfloor+h+1$, and the symbol $\doteq$ denotes equality up to a positive constant factor.

Since Wendland's functions are compactly supported, the interpolation matrices can be made sparse by appropriately scaling the support of the basic function. In the following we can consider only Wendland's functions depending on a shape parameter $\delta \in \mathbb{R}^{+}$. We list some of 
the most commonly used functions in $\mathbb{R}^{2}$ along with their degree of smoothness, i.e.

$$
\begin{aligned}
& \varphi_{2,0}(r) \doteq(1-\delta r)_{+}^{2} \\
& \varphi_{2,1}(r) \doteq(1-\delta r)_{+}^{4}(4 \delta r+1) \\
& \varphi_{2,2}(r) \doteq(1-\delta r)_{+}^{6}\left(35(\delta r)^{2}+18 \delta r+3\right) \\
& \varphi_{2,3}(r) \doteq(1-\delta r)_{+}^{8}\left(32(\delta r)^{3}+25(\delta r)^{2}+8 \delta r+1\right) .
\end{aligned}
$$

We remark that the functions $\varphi_{2, k}, k=0,1,2,3$, are strictly positive definite and radial not only on $\mathbb{R}^{2}$ but also on $\mathbb{R}^{m}$, for $m \leq 3$ (see [24]).

Referring to the image registration context we can define bivariate Wendland's transformations as follows.

Definition 1.Given a set of source landmark points $\mathscr{S}_{N}=\left\{\mathbf{x}_{j} \in \mathbb{R}^{2}, j=1,2, \ldots, N\right\}$, with associated the corresponding set of target landmark points $\mathscr{T}_{N}=\left\{\mathbf{t}_{j} \in \mathbb{R}^{2}, j=1,2, \ldots, N\right\}, \quad$ a Wendland's transformation $\mathbf{F}: \mathbb{R}^{2} \rightarrow \mathbb{R}^{2}$ is such that each its component

$$
F_{k}: \mathbb{R}^{2} \rightarrow \mathbb{R}, \quad k=1,2
$$

assumes the following form

$F_{k}(\mathbf{x})=F_{k}\left(x_{1}, x_{2}\right)=\sum_{j=1}^{N} c_{j k} \varphi_{2, h}\left(\left\|\boldsymbol{x}-\boldsymbol{x}_{j}\right\|_{2}\right)$,

with $\mathbf{x}=\left(x_{1}, x_{2}\right)$ and $\mathbf{x}_{j}=\left(x_{j 1}, x_{j 2}\right) \in \mathbb{R}^{2}$.

From Definition 1 it follows that the transformation function $F_{k}: \mathbb{R}^{2} \rightarrow \mathbb{R}$ is calculated for each $k=1,2$, and the coefficients $c_{j k}$ in (7) is to be obtained by solving two systems of linear equations.

\subsection{Wu's functions}

Another way to construct strictly positive definite radial functions with compact support is due to $\mathrm{Wu}$ [25]. He starts with the function

$$
\psi(r)=\left(1-r^{2}\right)_{+}^{s}, \quad s \in \mathbb{N},
$$

which is not strictly positive definite and radial on $\mathbb{R}^{m}$. Nevertheless, using convolution Wu obtains the function

$$
\begin{aligned}
\psi_{s}(r)=(\psi * \psi)(2 r) & =\int_{-\infty}^{+\infty}\left(1-t^{2}\right)_{+}^{s}\left[1-(2 r-t)^{2}\right]_{+}^{s} d t \\
& =\int_{-1}^{1}\left(1-t^{2}\right)_{+}^{s}\left[1-(2 r-t)^{2}\right]_{+}^{s} d t
\end{aligned}
$$

which is strictly positive definite and radial on $\mathbb{R}$. Moreover, the function $\psi_{s}$ is a polynomial of degree $4 s+1$ on its support, such that $\psi_{s} \in C^{2 s}(\mathbb{R})$.

Now, a family of strictly positive definite radial functions is constructed by the operator $\mathscr{D}$, namely $(\mathscr{D} \psi)(r)=-\frac{1}{r} \psi^{\prime}(r)$.
Definition 2. With $\psi_{s}(r)=\left[\left(1-.^{2}\right)_{+}^{s} *\left(1-.^{2}\right)_{+}^{s}\right](2 r)$ we define

$$
\psi_{k, s}=\mathscr{D}^{k} \psi_{s} .
$$

The functions $\psi_{k, s}$ are strictly positive definite and radial on $\mathbb{R}^{m}$ for $m \leq 2 k+1$, and are polynomials of degree $4 s-2 k+1$ on their support. Moreover, while in the interior of the support $\psi_{s} \in C^{2(s-k)}$, on the boundary the smoothness increases and $\psi_{s} \in C^{2 s-k}$. Then, we can consider the following Wu's functions along with their smoothness degree, i.e.

$$
\begin{aligned}
& \psi_{0,3}(r) \doteq(1-\delta r)_{+}^{7}\left(5+35 \delta r+101(\delta r)^{2}\right. \\
& \left.+147(\delta r)^{3}+101(\delta r)^{4}+35(\delta r)^{5}+5(\delta r)^{6}\right), \quad \mathrm{C}^{6} \\
& \psi_{1,3}(r) \doteq(1-\delta r)_{+}^{6}\left(6+36 \delta r+82(\delta r)^{2}\right. \\
& \left.+72(\delta r)^{3}+30(\delta r)^{4}+5(\delta r)^{5}\right) \text {, } \\
& \psi_{2,3}(r) \doteq(1-\delta r)_{+}^{5}\left(8+40 \delta r+48(\delta r)^{2}\right. \\
& \left.+25(\delta r)^{3}+5(\delta r)^{4}\right) \text {, } \\
& \psi_{3,3}(r) \doteq(1-\delta r)_{+}^{4}\left(16+29 \delta r+20(\delta r)^{2}+5(\delta r)^{3}\right) \text {. }
\end{aligned}
$$

Thus, concerning the image registration context we can consider the following definition, which involves Wu's transformations.

Definition 3.Given a set of source landmark points $\mathscr{S}_{N}=\left\{\mathbf{x}_{j} \in \mathbb{R}^{2}, j=1,2, \ldots, N\right\}$, with associated the corresponding set of target landmark points $\mathscr{T}_{N}=\left\{\mathbf{t}_{j} \in \mathbb{R}^{2}, j=1,2, \ldots, N\right\}$, a Wu's transformation $\mathbf{W}: \mathbb{R}^{2} \rightarrow \mathbb{R}^{2}$ is such that each its component

$$
W_{k}: \mathbb{R}^{2} \rightarrow \mathbb{R}, \quad k=1,2,
$$

assumes the following form

$$
W_{k}(\mathbf{x})=W_{k}\left(x_{1}, x_{2}\right)=\sum_{j=1}^{N} c_{j k} \psi_{s, 3}\left(\left\|\boldsymbol{x}-\boldsymbol{x}_{j}\right\|_{2}\right),
$$

with $\mathbf{x}=\left(x_{1}, x_{2}\right)$ and $\mathbf{x}_{j}=\left(x_{j 1}, x_{j 2}\right) \in \mathbb{R}^{2}$.

This means that the transformation function $W_{k}: \mathbb{R}^{2} \rightarrow \mathbb{R}$ is calculated for each $k=1,2$, and the coefficients $c_{j k}$ are to be obtained by solving two systems of linear equations.

\subsection{Gneiting's functions}

Starting with Wendland's functions and applying the turning bands operator, Gneiting in 2002 obtained a family of compactly supported functions [11]. Following [7], we can start with a function $\varphi_{m}$ that is strictly positive definite and radial on $\mathbb{R}^{m}$ for $m \geq 3$, and applying the turning bands operator results

$$
\varphi_{m-2}(r)=\varphi_{m}(r)+\frac{r \varphi_{m}^{\prime}(r)}{m-2}
$$

which is strictly positive definite and radial on $\mathbb{R}^{m-2}$. For example, starting with the Wendland function 
$\varphi_{4,1}(r)=(1-r)_{+}^{l+1}[(l+1) r+1]$ and applying the turning bands operator we obtain the functions

$$
\tau_{2, l}(r)=(1-r)_{+}^{l}\left(1+l r-\frac{(l+1)(l+4)}{2} r^{2}\right),
$$

which are strictly positive definite and radial on $\mathbb{R}^{2}$ provided $l \geq 7 / 2$. We list some specific functions from this family for various choices of $l$. All of the functions are in $C^{2}(\mathbb{R})$.

$$
\begin{aligned}
\tau_{2,7 / 2}(r) & \doteq(1-\delta r)_{+}^{7 / 2}\left(1+\frac{7}{2} \delta r-\frac{135}{8}(\delta r)^{2}\right), & & \mathrm{C}^{2} \\
\tau_{2,5}(r) & \doteq(1-\delta r)_{+}^{5}\left(1+5 \delta r-27(\delta r)^{2}\right), & & \mathrm{C}^{2} \\
\tau_{2,15 / 2}(r) & \doteq(1-\delta r)_{+}^{\frac{15}{2}}\left(1+\frac{15}{2} \delta r-\frac{391}{8}(\delta r)^{2}\right), & & \mathrm{C}^{2} \\
\tau_{2,12}(r) & \doteq(1-\delta r)_{+}^{12}\left(1+12 \delta r-104(\delta r)^{2}\right) . & & \mathrm{C}^{2}
\end{aligned}
$$

With regard to the image registration context we can define Gneiting's transformations as follows.

Definition 4.Given a set of source landmark points $\mathscr{S}_{N}=\left\{\mathbf{x}_{j} \in \mathbb{R}^{2}, j=1,2, \ldots, N\right\}$, with associated the corresponding set of target landmark points $\mathscr{T}_{N}=\left\{\mathbf{t}_{j} \in \mathbb{R}^{2}, j=1,2, \ldots, N\right\}, \quad$ a Gneiting's transformation $\mathbf{G}: \mathbb{R}^{2} \rightarrow \mathbb{R}^{2}$ is such that each its component

$$
G_{k}: \mathbb{R}^{2} \rightarrow \mathbb{R}, \quad k=1,2,
$$

assumes the following form

$$
G_{k}(\mathbf{x})=G_{k}\left(x_{1}, x_{2}\right)=\sum_{j=1}^{N} c_{j k} \tau_{2, l}\left(\left\|x-x_{j}\right\|_{2}\right),
$$

with $\mathbf{x}=\left(x_{1}, x_{2}\right)$ and $\mathbf{x}_{j}=\left(x_{j 1}, x_{j 2}\right) \in \mathbb{R}^{2}$.

As for Wendland's transformation, from Definition 4 it follows that the transformation function $G_{k}: \mathbb{R}^{2} \rightarrow \mathbb{R}$ is calculated for each $k=1,2$, and the coefficients $c_{j k}$ are to be obtained by solving two systems of linear equations.

We point out that in scattered data interpolation, in some cases, accuracy achieved using Gneiting's functions is better than that obtained using Wendland's functions (see [7]).

\section{Numerical experiments}

In this section, we compare the performances of the above methods when they are applied to give image transformations. In particular, we consider $C^{2}$ CSRBFs given by Wendland's (We2), Wu's (Wu2) and Gneiting's (Gn2) transformations. Moreover, to get a more complete picture, we show also the registration results obtained by Gaussian (G) and thin plate spline (TPS), which are widely used in this context.

In order to test the different CSRBF transformation schemes, we obtain several numerical results on some test cases. Here, for brevity, we refer only to two examples in $[10,15]$, which simulate typical medical cases where image portions scale or shift. These image portions represent rigid objects embedded in elastic material changing their position or form. The approach we propose can cope with local differences between corresponding images. In general these differences may be caused by the physical deformation of human tissue due to surgeries or pathological processes such as tumor growth or tumor resection. However, the aim is here to determine a transformation function, which connects the points of the source and target images, so that the target image is affected by the slightest possible deformation. This study and the related comparisons among the different transformation functions are made, in the test cases, also analyzing the behaviour of the root mean squares error (RMSE), which is obtained by computing the distances between the displacements of grid points $\mathbf{x} \in \mathscr{X}$ and the transformed values. It assumes the following form

$$
\mathrm{RMSE}=\sqrt{\frac{\sum_{\mathbf{x} \in \mathscr{X}}\|\mathbf{x}-\mathbf{F}(\mathbf{x})\|_{2}^{2}}{\sum_{\mathbf{x} \in \mathscr{X}} 1}},
$$

where $\|\cdot\|_{2}$ is the Euclidean norm.

Finally, we present some experimental results obtained by applying Gaussian, thin plate spline, Wendland's, Wu's and Gneiting's functions to real image data. More precisely, we consider two X-ray images of the cervical of an anonymous patient taken at different times. The considered real example is very similar to that taken by Modersitzki in [18].

\subsection{Test example 1: square shift and scaling}

We denote by $\mathscr{X}$ the set of $40 \times 40$ corner points of a regular grid superimposed on the source images (see Figure 1). The grid is transformed using 64 (Cases 1 and 2: square shift and scaling, respectively) landmarks and, in the case of square shift, also 4 quasi-landmarks, i.e. landmarks which have the same positions in both source and target images, to prevent an overall shift. The choice of considering two test cases with pair landmark sets is justified by the necessity of testing the interpolation schemes on different situations. In both cases, source and target image landmarks, shown in the images in Figure 1, are marked by a circle $(\circ)$ and a star $(\star)$, respectively.

Thus, to verify the goodness of the considered CSRBF transformations, we make a comparison of registration results using also Gaussian and thin plate spline transformations. More precisely, in Table 5.1 we report the RMSEs obtained by choosing fixed values of the shape parameters, i.e. taking $\alpha=2.0$ and $\delta=0.1$. These values are suitably selected in order to have a good compromise between accuracy and stability.

A complete error analysis is, in general, quite difficult since all the considered interpolation schemes are known 

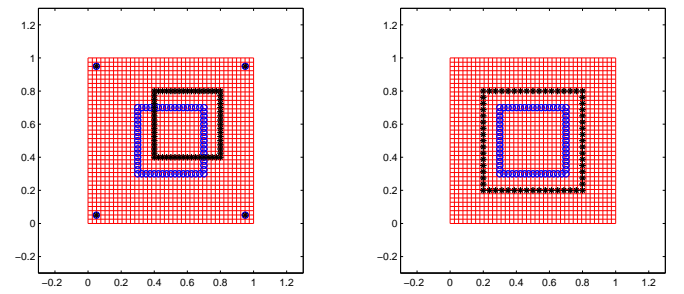

Fig. 1: Source and target landmarks: square shift (Case 1, left) and square scaling (Case 2, right).

Table 5.1:RMSEs for values of $\alpha=2.0$ and $\delta=0.1$.

\begin{tabular}{|c|c|c|}
\hline & Case 1 & Case 2 \\
\hline \hline $\mathrm{G}$ & $1.3768 \mathrm{E}-1$ & $2.0206 \mathrm{E}-1$ \\
\hline $\mathrm{TPS}$ & $1.0310 \mathrm{E}-1$ & $2.0929 \mathrm{E}-1$ \\
\hline $\mathrm{We} 2$ & $1.0911 \mathrm{E}-1$ & $1.8856 \mathrm{E}-1$ \\
\hline $\mathrm{Wu} 2$ & $1.0924 \mathrm{E}-1$ & $1.9318 \mathrm{E}-1$ \\
\hline $\mathrm{Gn} 2, l=5$ & $1.0911 \mathrm{E}-1$ & $1.6670 \mathrm{E}-1$ \\
\hline $\mathrm{Gn} 2, l=7 / 2$ & $1.0039 \mathrm{E}-1$ & $1.8307 \mathrm{E}-1$ \\
\hline
\end{tabular}

to be accurate and, in fact, all errors are of the same orders of magnitude. However, the RMSEs point out that in Case 1 the best result is obtained by using Gneiting's CSRBF with $l=7 / 2$, whereas in Case 2 the lowest error is still given by Gneiting's function with $l=5$. The goodness of Gneiting's transformations is also confirmed by a graph standpoint (see Figures 2-3), where shape and smoothness of the different transformed grids are shown. In such figures, for brevity, we report a restricted number of registration results obtained by applying CSRBF-based interpolation schemes and, for comparison, the most accurate result that is given by either Gaussian or TPS transformations.

We note that irregular grids may be obtained using Gaussian, while smoother results are given by thin plate spline and compactly supported radial basis functions. Finally, we point out that the condition numbers of interpolation matrices generated by Gaussian might turn out quite large in both cases, namely $10^{17} \div 10^{19}$.

\subsection{Test example 2: circle contraction and expansion}

In this subsection we consider a radial transformation, that is, a circle contraction. It may be viewed as a very schematic model for a tumor resection in surrounding elastic brain tissue. In this model (see [15]) the outer circle corresponds to the skull bone, which is assumed to be rigid. The inner circle represents the boundary of the

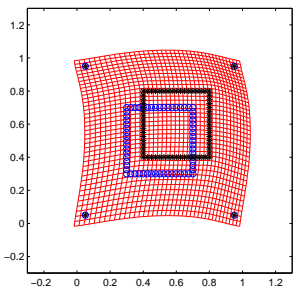

(a) TPS

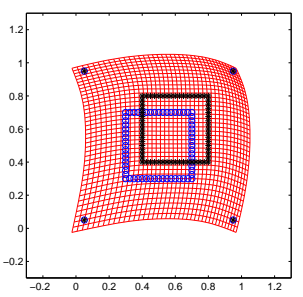

(c) We2

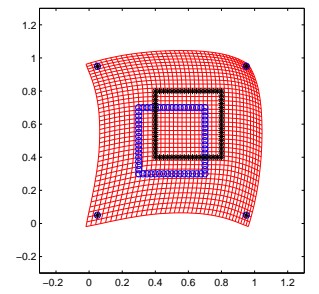

(b) $\mathrm{Gn} 2, l=5$

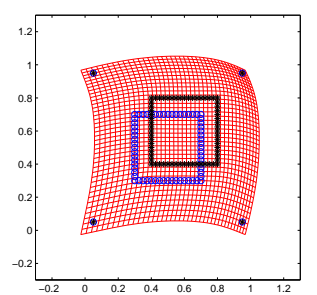

(d) Wu2
Fig. 2: Case 1: registration results for the shift of a square using $\delta=0.1$.

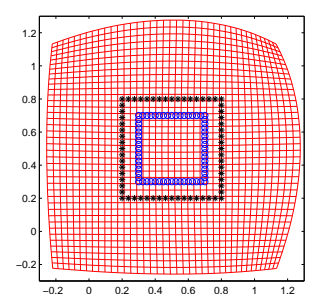

(a) $\mathrm{G}$

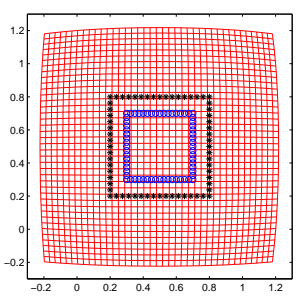

(c) We2

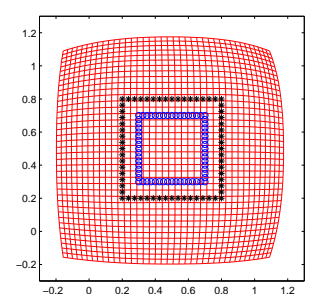

(b) $\mathrm{Gn} 2, l=5$

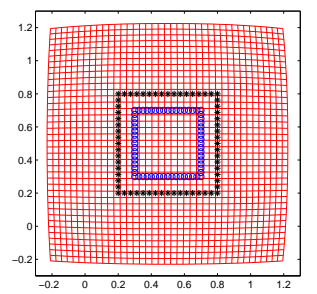

(d) $\mathrm{Wu} 2$
Fig. 3: Case 2: registration results for the scaling of a square using $\alpha=2.0$ and $\delta=0.1$.

tumor, whereas the space between the inner and the outer circle is assumed to be filled with elastic material, which corresponds to brain tissue.

The grids are transformed using 20 equidistant landmarks placed on the inner circle and, to prevent an overall shift, also 40 quasi-landmarks, i.e. landmarks at invariant positions, at the outer circle in the source and 
target images. These point-landmarks, shown in Figure 4 (a), are marked by a circle (o) and a star $(\star)$, respectively.

In these experiments we compare at first the registration results obtained by using We2 and Gn2 with $l=7 / 2$ as CSRBFs and TPS for a comparison. In particular, Figure 4 (b)-(d) shows registration results using $\delta=0.1$ as a shape parameter, whereas the related errors are reported in Table 1.

Table 1: RMSEs for values of $\alpha=2.0$ and $\delta=0.1$.

\begin{tabular}{|c|c|}
\hline & Case 3 \\
\hline \hline $\mathrm{G}$ & $1.2287 \mathrm{E}-1$ \\
\hline $\mathrm{TPS}$ & $7.7354 \mathrm{E}-2$ \\
\hline $\mathrm{We} 2$ & $9.1792 \mathrm{E}-2$ \\
\hline $\mathrm{Wu} 2$ & $9.4400 \mathrm{E}-2$ \\
\hline $\mathrm{Gn} 2, l=5$ & $7.9795 \mathrm{E}-2$ \\
\hline $\mathrm{Gn} 2, l=7 / 2$ & $5.2041 \mathrm{E}-2$ \\
\hline
\end{tabular}

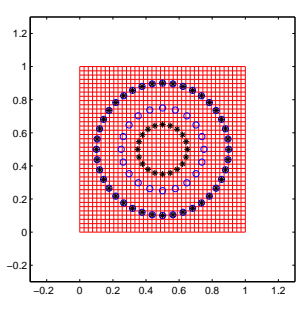

(a) Source-target landmarks

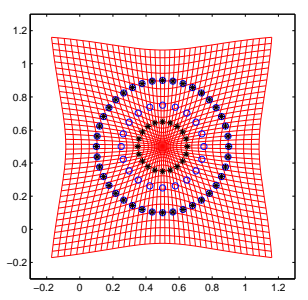

(c) $\mathrm{We} 2$

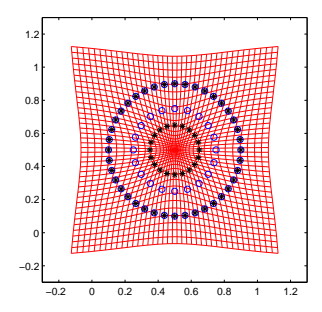

(b) TPS

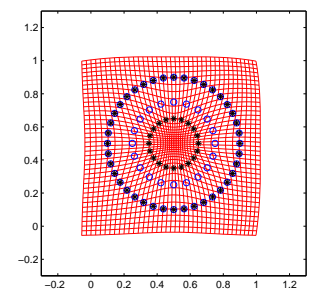

(d) Gn2, $l=7 / 2$
Fig. 4: Source and target landmarks and registration results for circle contraction using $\delta=0.1$.

Also in this case errors are less and smoother transformed images are obtained when Gneiting's transformations are employed, especially with $l=7 / 2$.

For the circle expansion we found very similar results to those achieved for square scaling and then we do not report them. However, we point out that also in this case the best registration results were obtained using $\mathrm{Gn} 2$, with $l=5$.

\subsection{Real-life case: an application to medical images}

In this subsection we present some experimental results obtained by applying Gaussians, thin plate splines, Wendland's, Wu's and Gneiting's functions to real image data. More precisely, we consider two X-ray images of the cervical of an anonymous patient taken at different times. The considered real example is very similar to that given by Modersitzki in [18]. In Figure 5 we show the two images along with landmarks and quasi-landmarks, setting on the left the source image and on the right the target one. The size of both images is $512 \times 512$ pixels. In particular, within each of the two images we have manually selected 6 landmarks within and, moreover, to fix transformation and to prevent an overall shift, we have added 12 quasi-landmarks on the boundaries of the source and target images.

Each result in Figure 6 (a)-(f) represents a transformed image, obtained using all the considered RBFs. In accordance with the test examples in Subsections 5.1 and 5.2, for the Gaussian we have used the parameter value $\alpha=2.0$, for Wendland's, Wu's and Gneiting's transformations the value is $\delta=0.1$. We observe that Gaussian transformation strongly deforms the image, while using TPS gives less significant deformations. Better registration results are obtained with CSRBFs, especially when we use the Gn2. Moreover, we point out that registration results are better if we use small values for the parameter, i.e. $\delta \in[0.1,0.5]$.
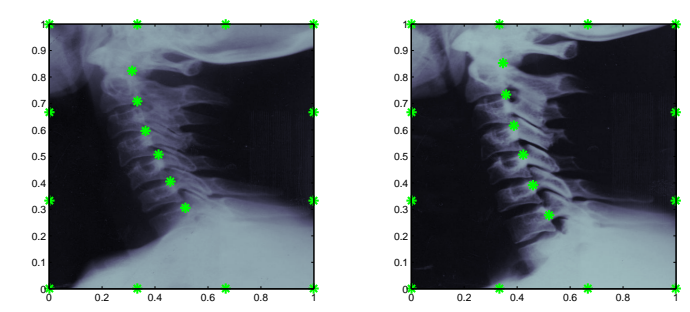

Fig. 5: Source and target cervical images with landmarks and quasi-landmarks (left to right).

\section{Conclusions}

In this paper we compared some well established interpolation methods and some techniques recently proposed in the context of image registration such as the 


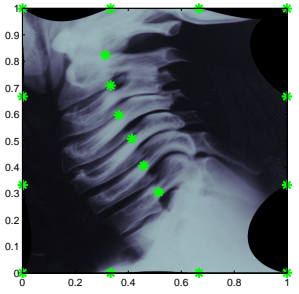

(a) G

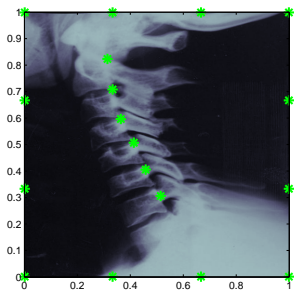

(c) We2

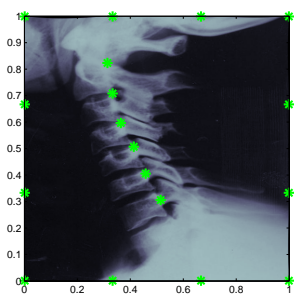

(e) $\mathrm{Gn} 2, l=5$

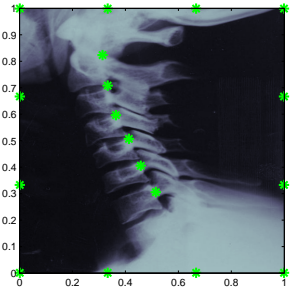

(b) TPS

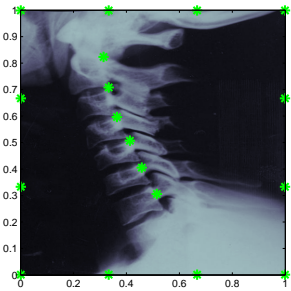

(d) $\mathrm{Wu} 2$

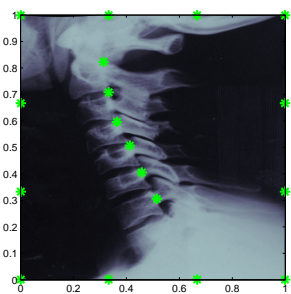

(f) $\mathrm{Gn} 2, l=7 / 2$
Fig. 6: Real-life case: registration results using $\alpha=2.0$ and $\delta=$ 0.1 .

CRBFs, specifically Wendland's, Wu's and Gneiting's functions. To this aim, we briefly recalled all the above local interpolation schemes and commented their performances in landmark-based image registration, taking into account the wide literature on the topic.

In particular, we were interested in pointing out which scheme may be preferable in a specific situation. Moreover, since a great number of techniques require the use of a shape parameter which might greatly influence registration results, we remark that sometimes may be necessary a compromise between accuracy and smoothness.

Here, we used interpolating transformations which accomplish an exact match of corresponding landmarks. This implicitly means that the landmark positions are exactly known. However, if we have to deal with landmark localization errors, then it would be advantageous to weaken the interpolation conditions by introducing an approximation scheme. Further investigations in this direction are still required and ongoing.

\section{Acknowledgements}

The authors gratefully acknowledge the financial support of the Department of Mathematics "G. Peano", University of Torino, project "Numerical analysis for life sciences" (2012).

\section{References}

[1] G. Allasia, R. Cavoretto, A. De Rossi, B. Quatember, W. Recheis, M. Mayr and S. Demertzis, In: T.E. Simos et al. (Eds.), Proceedings of the ICNAAM 2010, AIP Conf. Proc., 1281, 716-719 (2010).

[2] G. Allasia, R. Cavoretto and A. De Rossi, Math. Methods Appl. Sci., 35, 923-934 (2012).

[3] F.L. Bookstein, IEEE Trans. Pattern Anal. Mach. Intell., 11, 567-585 (1989).

[4] R. Cavoretto and A. De Rossi, In: T.E. Simos et al. (Eds.), Proceedings of the ICNAAM 2008, AIP Conf. Proc., 1048, 970-973 (2008).

[5] R. Cavoretto, A. De Rossi and B. Quatember, J. Numer. Anal. Ind. Appl. Math., 5, 141-152 (2011).

[6] R. Cavoretto and A. De Rossi, In: T.E. Simos et al. (Eds.), Proceedings of the ICNAAM 2012, AIP Conf. Proc., 1479, 1335-1338 (2012).

[7] G.E. Fasshauer, Meshfree Approximation Methods with MATLAB, World Scientific Publishers, Singapore, (2007).

[8] B. Fischer and J. Modersitzki, Inverse Probl., 24, 16 (2008).

[9] J.M. Fitzpatrick, D.L.G. Hill and C.R. Maurer Jr, In: M. Sonka, J.M. Fitzpatrick (Eds.), Handbook of Medical Imaging. Medical Image Processing and Analysis, SPIE Press, Bellingham, 2 447-513 (2000).

[10] M. Fornefett, K. Rohr and H.S. Stiehl, Image Vision Comput., 19, 87-96 (2001).

[11] T. Gneiting, J. Multivariate Anal., 83 (2002).

[12] A. Goshtasby, 2-D and 3-D Image Registration, Wiley, New York, (2005).

[13] J.V. Hajnal, D.L.G. Hill and D.J. Hawkes, Medical Image Registration, CRC Press, Baton Raton, Florida, (2001).

[14] M. Holden, IEEE Trans. Med. Imaging, 27, 111-128 (2008).

[15] J. Kohlrausch, K. Rohr and H.S. Stiehl, J. Math. Imaging Vision, 23, 253-280 (2005).

[16] Medical Image Processing, Analysis and Visualization (MIPAV), software package, http://mipav.cit.nih.gov.

[17] J. Modersitzki, Numerical Methods for Image Registration, Oxford Univ. Press, Oxford, (2004).

[18] J. Modersitzki, FAIR: Flexible Algorithms for Image Registration, Fundam. Algorithms, SIAM, Philadelphia, PA, 6, (2009).

[19] J.P.W. Pluim, J.B.A. Maintz and M.A. Viergever, IEEE Trans. Med. Imaging, 22, 986-1004 (2003).

[20] B. Quatember, M. Mayr, W. Recheis, S. Demertzis, G. Allasia, A. De Rossi, R. Cavoretto and E. Venturino, Math. Comput. Simulation, 81, 608-622 (2010).

[21] B. Quatember, M. Mayr, W. Recheis, S. Demertzis, G. Allasia, A. De Rossi, R. Cavoretto and E. Venturino, In: R. Moreno-Díaz et al. (Eds.), Computer Aided Systems Theory - EUROCAST 2011, LNCS, Springer-Verlag Berlin Heidelberg, 6928, 248-255 (2012). 
[22] K. Rohr, Landmark-Based Image Analysis, Using Geometric and Intensity Models, Kluwer Academic Publishers, Norwell, MA, (2001).

[23] O. Scherzer, Mathematical Models for Registration and Applications to Medical Imaging, Springer, New York, (2006).

[24] H. Wendland, Scattered Data Approximation, Cambridge University Press, Cambridge, (2005).

[25] Z. Wu, Adv. Comput. Math., 4, 283-292 (1995).

[26] B. Zitová and J. Flusser, Image Vision Comput., 21, $977-$ 1000 (2003).

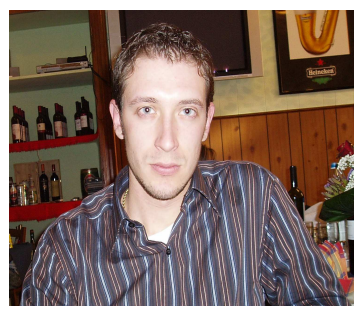

Roberto Cavoretto is a Fixed-Term Research Fellow of Numerical Analysis at the University of Torino, Italy. He got his Ph.D. degree in Science and High Technology (Mathematics) at the University of Torino in 2010. In the same university he held a post-doc position until 2011. His research activity is mainly focused on topics of numerical analysis and applied mathematics such as: scattered data approximation, spherical interpolation, and applications to image registration. He has published research articles in international refereed journals of applied mathematics.

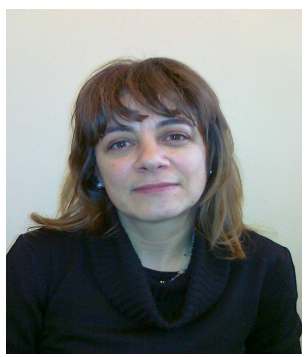

Alessandra De Rossi
is Assistant Professor
of Numerical Analysis
at the University of Torino.
She received the PhD
degree in "Computational
Mathematics" at the
University of Milano
(Italy) in 1997. She has
published research articles in
international journals of pure

and applied mathematics. She is referee of several international journals in the frame of numerical analysis and applied mathematics. Her main research interests are: meshfree approximation, fast algorithms for scattered data interpolation, image registration. 